\title{
BMJ
}

\section{Women's views about management and cause of urinary tract infection: qualitative interview study}

\author{
G M Leydon, senior researcher and NIHR fellow, ${ }^{1} \mathrm{~S}$ Turner, senior research fellow, ${ }^{2} \mathrm{H}$ Smith, chair in primary \\ care, ${ }^{3} \mathrm{P}$ Little, professor of primary care research ${ }^{1}$ on behalf of the UTIS team
}

${ }^{1}$ Primary Medical Care, Community Clinical Sciences Division,

University of Southampton School of Medicine, Aldermoor Health Centre, Southampton S016 5ST

${ }^{2} \mathrm{NIHR}$ Public Health Research Programme NETSCC, Alpha

House, University of Southampton Science Park, Southampton S016 7NS

${ }^{3}$ Brighton and Sussex Medical School, University of Brighton, Brighton BN1 9PH

Correspondence to: G M Leydon gerry@soton.ac.uk

Cite this as: $B M J$ 2010;340:c279 doi:10.1136/bmi.c279

\section{ABSTRACT}

Objectives To explore the views of women with urinary tract infection on the acceptability of different strategies for managing the infection, including delayed use of antibiotics, and the cause of infection.

Design Qualitative interview study with semistructured one to one interviews within a randomised controlled trial of different management strategies. Analysis drew on some of the principles of constant comparison to generate key themes grounded in reported experiences and understandings.

Setting Seven general practices across four counties in southern England.

Participants 21 women presenting to general practices who were taking part in the larger trial.

Results Women preferred not to take antibiotics and were open to alternative management approaches. With a strategy of "antibiotic delay" some women felt a lack of validation or that they were not listened to by their general practitioner. Women attributed urinary tract infection to lifestyle habits and behaviours, such as poor hygiene, general "negligence," and even a "penalty of growing old." Conclusion A clear acknowledgment of women's triggers to consult is needed. If women are asked to delay taking antibiotics, the clinician must address the particular worries that women might have and explain the rationale for not using antibiotics immediately.

\section{INTRODUCTION}

Acute urinary tract infection is one of the commonest acute bacterial infections among women. ${ }^{1-3}$ Most women presenting in primary care are prescribed an antibiotic. Conventional courses of antibiotics probably help to resolve symptoms but are also likely to have side effects, ${ }^{45}$ including skin rash, ${ }^{6}$ vaginal symptoms, ${ }^{7}$ and other side effects. ${ }^{8}$ The strategy of universal use of antibiotics in these women is being questioned, ${ }^{9-11}$ in part because the condition is often self limiting and non-pharmacological alternativessuch as the use of cranberry juice, teas, or herbal remedies and potassium citrate or sodium biocarbonateexist, although the evidence for effectiveness is weak. $^{1213}$ The potential effectiveness of antibiotics must also be balanced against wider issues for the National Health Service (NHS), particularly the mounting concerns about increasing workload for self limiting illness ${ }^{2}$ and the growing problem of antibiotic resistance. ${ }^{1415}$ The use of diagnostic techniques and antibiotics might encourage belief in antibiotics and in the necessity of seeing a general practitioner for the problem, thus "medicalising" a condition that is often self limiting. ${ }^{1617}$ This leads to greater antibiotic use and increased antibiotic resistance. ${ }^{18-20}$

Given these concerns and the limited evidence base for alternative management options, there was a need for trials to allow estimation of the advantages and disadvantages of antibiotics, ${ }^{21}$ antibiotic strategies, and non-antibiotic alternatives. We conducted a pragmatic randomised controlled trial to test several management strategies, including antibiotic delay. ${ }^{22}$ Qualitative interviews nested in the trial explored interviewees' attitudes towards antibiotics, their experience of a delayed antibiotic prescription, and their views on the cause of urinary tract infection.

\section{METHODS}

Participants and procedure

Participants were recruited from a large randomised trial of different management strategies, in which patients were randomised within the consultation to one of five management groups: empirical antibiotic treatment; empirical delayed antibiotics; antibiotic targeted by symptom score (two or more of urine cloudy, urine with offensive smell, moderately severe dysuria, or nocturia); antibiotic according to dipstick algorithm (nitrites or leucocytes and a trace of blood); or midstream urine analysis with symptomatic treatment until culture and sensitivity results were available and then antibiotics targeted according) (box 1).

To be eligible for inclusion participants had to be taking part in the larger trial, have consented to have a single face to face interview, and have been allocated to a management group in which delayed antibiotics were specified by the protocol, meaning this option was discussed and negotiated flexibly with each patient, and they were given access to the delayed prescription at any stage. The third criterion ensured that we were able to explore participants' thoughts on the appropriate treatment of urinary tract infection and their views on the acceptability or otherwise of being 


\section{Box 1 Management strategies representing common approaches in general practice}

\section{Empirical antibiotic treatment}

This is the most common strategy in practice and was used as the control group. Patients were prescribed an antibiotic (trimethoprim $200 \mathrm{mg}$ twice daily) for three days. If patients were allergic to trimethoprim they were offered an alternative (cefaclor or cefalexin) as this was not a trial of antibiotics per se but a trial of management/advice strategies.

\section{Empirical delayed antibiotics}

All patients were advised to drink plenty and offered a delayed antibiotic prescription to be used if symptoms did not start to improve after 48 hours (doctors were asked to leave a prescription at the front desk for patients to collect as necessary, or they could negotiate with the patient if they wanted to take the prescription away). The rationale is that $40 \%$ of patients with suspected urinary tract infection do not have infection, and, even in those with laboratory diagnosed infections, the illness is likely to be self limiting.

\section{Symptom score}

Patients who had two or more of urine cloudy on examination, urine with an offensive smell on examination, patient's report of moderately severe dysuria, or patient's report of moderately severe nocturia were offered immediate antibiotics-that is, symptomatic treatment only. From a previous study we estimated the sensitivity of this approach as $68 \%,{ }^{18}$ so patients without two or more features were also offered a delayed antibiotic prescription to use if their symptoms were not settling after a few days.

\section{Dipstick}

Patients who had either nitrites or leucocytes and a trace of blood were offered antibiotics initially. Patients not fulfilling this criterion (which we estimate had a sensitivity of $71 \%$ ) were offered a delayed antibiotic prescription to use if their symptoms were not settling after a few days

\section{Midstream urine analysis}

This was the only group in which a midstream urine sample was routinely collected. Patients were offered symptomatic treatment until the results of the test were known. This is the "reference" method of diagnosing infection and of targeting antibiotic use.

asked to delay taking antibiotic medication. At interview it was clear that seven of the interviewees had in fact received antibiotic medication. We interviewed general practitioners in cases where the policy of delay was apparently not followed, and they reported patients' expectations as a prominent reason for negotiating a different strategy.

Patients were drawn from practices across Berkshire (Reading), Wiltshire (Salisbury), Hampshire (Romsey, Portsmouth, Waterlooville, Havant), and Dorset (Dorchester). GML and ST conducted the interviews in women's homes. Each interview lasted an hour on average and was audio-taped and transcribed verbatim by a professional freelance transcriptionist. GML checked 10 full transcriptions against corresponding audio-recorded interviews and found good accuracy. Subsequent quality checks were made during analysis.

\section{The interviews}

A semistructured topic guide ensured that critical topics were covered in every interview, while also providing the necessary flexibility to allow participants to volunteer topics that were germane to them. The interviews were designed to elicit participants' experiences and understanding of urinary tract infection, their beliefs regarding treatment, and their views about the management strategy of delayed antibiotic prescribing (that is, a prescription being available at reception (or, by negotiation with the general practitioner, that could be taken away) for use should symptoms not start to settle).

\section{Analysis}

Following principles of constant comparison, we thematically analysed transcribed interview data in an iterative manner. This involved moving back and forth between interview transcripts, early analytical memos/notes about process, and the research literature. ${ }^{2324}$ Vertical and horizontal familiarisation of the interviews was aided by production of summaries of each. ${ }^{25}$ After repeated readings (while listening to taped interviews) GML developed an early coding framework based on five transcripts. "Crude counts" of observations/themes provided an indication of their frequency. ${ }^{23}$ In a sample of transcribed interviews, PL independently checked the validity of the early codes and the accuracy/reliability of their application to the transcribed data. The consistency of coding/interpretation was also checked during analysis by revisiting annotated transcripts at different time periods. Codes were iteratively developed by all authors and eventually all data were organised and codes merged to generate themes that captured the range of experiences and views reported.

\section{RESULTS}

\section{Participants}

Thirty three women were approached to take part in the interview study. Twenty seven agreed and 21 (aged 21-64, median 40) were interviewed before data saturation was reached (one tape failure meant that 20 interviews were available for analysis). Reasons for refusal included being too busy or unavailable in working hours. Refusals for both the trial and then for the qualitative study were low. The characteristics of women participating in the qualitative study were similar to the overall trial cohort: married $65 \%$ v 73\%, past cystitis $88 \%$ v $85 \%$, number of medical concurrent problems $3.0 v 2.6$, age leaving education $17.6 v 17.6$, severity of frequency of urination symptoms at baseline $3.5 v 3.5$, respectively.

\section{Patients' experiences}

In their interviews patients traced their experiences from the onset of symptoms and their attempts to self manage, through to their final decision to attend a general practitioner. ${ }^{26}$ Many described their initial reluctance to attend their general practitioner. In most cases the severity or duration of symptoms, or both, eventually prompted a visit (tables 1 and 2).

Patients' views about the management of urinary tract infection

Of the 20 interviews recorded, 13 women were asked to delay taking antibiotics and of these 10 viewed the strategy positively (patients 2, 3, 5, 6, 7, 9, 12, 14, 15, 20) and three (patients $8,11,17$ ) reported negative experiences. The preponderance of positive reports about the experience of delayed medication or having 
Table 1|Summary of volunteered signs and symptoms experienced by women with urinary tract infection (verbatim)

\begin{tabular}{ll}
$\begin{array}{l}\text { Symptom/sign } \\
\text { Physical }\end{array}$ & $\begin{array}{c}\text { No of women who } \\
\text { mentioned it }\end{array}$ \\
\hline Frequency & 8 \\
\hline Very painful/severe/bad & 7 \\
\hline Bleeding & 6 \\
\hline Cold/flu-like symptoms/raised temperature & 4 \\
\hline Back ache/pain & 3 \\
\hline Stinging/burning/stabbing & 3 \\
\hline Pains/balloons in tummy & 3 \\
\hline Uncomfortable & 3 \\
\hline Poor concentration & 2 \\
\hline Pain when urinating & 2 \\
\hline Smelly urine & 2 \\
\hline Tired/exhausted & 2 \\
\hline Sleepless & 1 \\
\hline Hot sensation in bladder & 1 \\
\hline Pain worsening & 1 \\
\hline General/emotional/functional & 3 \\
\hline Generally unwell/lousy/poorly & 6 \\
\hline Normal duties disrupted/debilitating & 3 \\
\hline
\end{tabular}

non-drug alternatives, or both, corresponded with participants' reported reticence about relying on antibiotics. Two interviewees expressed a preference for antibiotic medication. One indicated that fear of worsening symptoms had prompted her to seek help in a preventative fashion and for her the optimal treatment was antibiotic medication (patient 7). The other's preference was based on her experience of successful treatment in the past.

Seven interviewees reported being prescribed antibiotic medication immediately (patients 1, 4, 10, 13, 16, 18, 19) (table 3).

\section{Positive experiences \\ Being offered an alternative to antibiotics}

Three of the interviewees reflected on their allocation to the "delay" arm of the trial in highly positive terms.

"She [the nurse] said ... . if you agree to take part then you'll have an envelope and you may be given antibiotics, you may not ... and I said well I really would rather not have antibiotics ... but luckily enough ... I got the cranberry juice [laughter] one ..." (patient 5) "I went in and I didn't want to take antibiotics so I was quite glad when he gave me this other [Uvacin, a herbal over the counter remedy]"(patient 12)

\section{Avoiding side effects of antibiotics}

Participants spoke about their initial reactions to antibiotic delay in terms that suggested a careful weighing of the consequences of immediate use versus delayed or no use.

"Well, I sort of sat there and went, oh, not three more days, but then when I thought about the side effects, it was like, well . . . do I really want the side effect [of thrush] ... then that's going to be another three, four days ... of more pain in the same area and I just thought, well ... you've had it for seven days and if you wait another three it will hopefully have gone anyway" (patient 14)

Most of the participants with experience of antibiotic use had developed thrush (one of the commonest side effects) as a consequence and this mediated their desire for antibiotic medication.

"I know that thrush can be a side effect of the antibiotic and I have suffered that in the past as well, so anything to avoid that situation" (patient 6)

Avoiding side effects was a strong driver for viewing antibiotic delay positively, as was a reported belief in and preference for "natural" alternatives.

"Antibiotics in general have caused me a quite severe rash on my legs ... I suppose that is one of the reasons but I don't think that's the prime reason. I think the main reason is that I just don't think it is right for the body to keep taking them" (patient 5)

\section{Having a general practitioner who provides "natural"} alternatives

Being offered an alternative to antibiotics was particularly well received by patients who indicated a belief in holistic medicine and wherever possible the avoidance of orthodox medicine.

"I really like the fact that my GP, who I mentally associate with y'know antibiotics and drugs of some sort, has suggested a herbal remedy and fruit juice ... I think it's great ... rather than trying to give me antibiotics straight away ... I'd love a practice that really combines all the kind of natural therapies ... whether it be herbal, homoeopathic, or through nutrition and diet" (patient 2)

"He didn't give me antibiotics, but I wouldn't have taken them anyway ... I'm not a great believer in antibiotics" (patient 15)

Despite being in pain, one woman's desire to avoid orthodox medication meant that delay was still viewed

Table 2 Duration of signs and symptoms experienced by women with signs/symptoms of urinary tract infection before they visit general practitioner

\begin{tabular}{lc} 
Time waited before consultation & Patient number \\
1 night and 1 day & 18 \\
\hline $2-3$ days & $8,11,12,13,15,17$ \\
\hline 4 days & 6 \\
\hline 7 days & $7,9,14,16,20$ \\
\hline 10 days & 4 \\
\hline 3 weeks & 2,19 \\
\hline 4 weeks & $3,5,10$
\end{tabular}




\begin{tabular}{|c|c|}
\hline Patient & Reason \\
\hline 4 & Interstitial cystitis (chronic inflammation of bladder wall) \\
\hline 10 & He didn't suggest waiting because "I was in such a state" \\
\hline 13 & She told the doctor what she wanted and did not delay or try Uvacin \\
\hline 16 & $\begin{array}{l}\text { No delay recommended, "she felt in this case, because l'd already ... tried other courses } \\
\text { of action and that was seven days and the symptoms were becoming more severe rather } \\
\text { than better, that antibiotics was probably the right course of action to take" }\end{array}$ \\
\hline 18 & Tried antibiotic and it did not work. Then had to try another type \\
\hline 19 & Given antibiotic immediately for immediate use \\
\hline
\end{tabular}

as appropriate. Her positive disposition was no doubt reinforced by her recovery without intervention.

"I was in quite a lot of pain, I thought, well, OK, I can wait, I'll give it, you know, I'll give it four or five days and then see if I get any better, and, and I did get better" (patient 9)

Having a "back-up" prescription and feeling validated Just one participant explicitly mentioned that the recommended delay was acceptable because of her "faith" in her general practitioner, "I have a great deal of faith in my GP . . . and because he was happy to suggest the Uvacin, I was happy to accept that" (patient 6).

Others who were offered a delayed antibiotic reported that they derived comfort from the knowledge that a prescription was available if required.

"I guess in the back of my mind there was a slight reassurance that if all else fails I've got it [the prescription]" (patient 6)

"I felt OK because I knew that the prescription was there if I needed it" (patient 20)

\section{Negative experiences}

Not feeling validated

Receiving a (delayed) antibiotic prescription contributed to participants' feelings that their symptoms had been validated and taken seriously. By contrast, the three participants who reported negative experiences of the delay strategy spoke of their concern that their knowledge had been ignored.

\section{"I'm quite willing to listen, but I know my own body" (patient 8)}

It was clear that the patients who reported negative experiences doubted whether their general practitioners had acknowledged their symptoms or accepted the legitimacy of their complaint.

"He really didn't want me to have [an antibiotic] he was a bit blasé about it and um ... I thought I told him ... I was sort of passing blood" (patient 11)

Feeling that their complaint was not viewed as legitimate by their general practitioner could be influenced by participants' knowledge that antibiotics had the potential to alleviate symptoms more rapidly compared with no antibiotic.
"[I could have] got rid of it a lot sooner and gone back to work, instead of missing time at work" (patient 11)

\section{Too late to wait}

For a few participants, delay had proved to be alarming because their symptoms had, in their opinion, "just gone past the waiting stage" (patient 8). Indeed, this particular participant did not delay, as recommended, because her symptoms were interfering with her work. She reported that she was in "terrible pain and it was frightening" and "I was nearly in tears." The seven participants in the delay arm of the trial who were offered immediate antibiotics had described their symptoms in similarly serious terms. Understanding women's experiences like these helped to explain why some participants did not feel validated or taken seriously - that is, a consultation that resulted in no antibiotic or a delayed antibiotic could symbolise a rejection of their symptoms.

Another interviewee who expressed dissatisfaction spoke about how she had already attempted self help measures and in this way had already delayed.

"I think probably at the time I just thought well I've waited this long, I've done all the help, self help measures myself, um ... the fact that he was asking (slight laughter) me to delay even longer was, oh, I don't want to do this but I think I did and then I think it got to the stage where I just thought no" (patient 17)

Thus, a general practitioner's recommendation to delay could carry the risk of invalidating the basis for a patient's consultation and the attempts they had made to self care before their attendance. Importantly, when faced with continuing symptoms women reported that they would prioritise knowledge of their "own bodies" and exercise their right to collect and use the "delayed" antibiotics or attend their general practitioner again.

\section{Causes of urinary tract infection}

All participants were asked what they thought caused urinary tract infection. Table 4 shows the range of causes volunteered by participants. Fifteen of the 20 women interviewed discussed previous experiences of urinary tract infection or cystitis, yet when asked to discuss the natural history/signs and symptoms of urinary tract infection most of the women struggled. Responses also indicated a need for increased opportunities for patients who attend with a suspected urinary tract infection to discuss the condition and the evidence (and uncertainty) about the effectiveness of antibiotic medication.

Lifestyle explanations were often cited as contributing to urinary tract infection. Women mentioned refraining from certain behaviours such as "drinking caffeine," "not drinking too much wine," and not being "negligent" when it comes to cleanliness. Embedded in most participants' reports was a "duty to stay healthy" and to live a life centred on the concept of self discipline (good diet, exercise, washing after sex, 
Table $4 \mid$ Causes of urinary tract infection according to women presenting to primary care* Authors' summaries or verbatim if shown in quotes

\begin{tabular}{|c|c|}
\hline Patient & What do you think causes a urinary tract infection? \\
\hline 1 & Wiping the wrong way, low immunity, and antibiotics \\
\hline 2 & $\begin{array}{l}\text { Once you have had it, tend to be more susceptible, sex, diet, and lifestyle. Wine and drinking } \\
\text { Bovril caused "mine" }\end{array}$ \\
\hline 3 & $\begin{array}{l}\text { Age, "drying up," "penalty of growing old." Long bicycle ride on holiday and sitting on damp } \\
\text { towel }\end{array}$ \\
\hline 4 & Deviant case; interstitial cystitis \\
\hline 5 & Dehydration, infection after diarrhoea, age, "have to be more careful as get older," stress \\
\hline 6 & Dehydration, alcohol, enhanced sexual activity, being on holiday \\
\hline 7 & "Don't know really"; drugs and antibiotics? People go through "phases" \\
\hline 8 & Dehydration, sex, perfumed products, "that's all I know" \\
\hline 9 & $\begin{array}{l}\text { "I'm not sure really"; "I think some of it is cleanliness" ... "I sit for [prolonged periods] and } \\
\text { [toilet breaks are very quick] ... You leave it until you have to go" }\end{array}$ \\
\hline 10 & $\begin{array}{l}\text { "I'm a bit perplexed about it 'cause it's something that I never had... growing up ... I've heard } \\
\text { that it's associated with the menopause." "I assume it can be caused by your sex life ... or } \\
\text { some irritation. I've been told that it's ... a germ and it can be caused by a germ in the water." } \\
\text { Individual cause: "doesn't seem to be any one particular cause" }\end{array}$ \\
\hline
\end{tabular}

11 A bug? Wiping the wrong way, tight clothes, "or am I just making that up." Individual: "I don't know in my case what [causes it], because I don't think l've been doing anything different ... to suddenly get it at my age [mid-50s] and not ever had it before ... I don't know"

12 "I've read loads on it; I should know [laughter from both]. I've had all the books out. Every time something goes wrong I read all the books . . . I don't know . . . it's . . I I don't know, I can't think of it now"

$13 \quad$ Active sex life

14 "I don't know . . . sometimes if I've . . . become sexually active, I always get cystitis . . . 100\% I can guarantee it. Hence I just don't bother anymore [laughter]. No, go away, because I know I'm going to get ... it tends to be around my period that I get it ... it's yeah, if I'm, if I'm, if I'm in a relationship, it's a, it's a definite [laughter] ... it's kind of inconvenient, but you know"

15 Individual cause: "I feel that it is actually just a bit of, well, a bit of bad luck and perhaps a little bit of lack of concentration [when going to the loo], because I do remember . . . being a little less careful than I should normally have been"; "negligence"; "the more stressed you are the more it ... makes your body vulnerable. I think it lowers your body's resistance in so many ways"

16 "People don't drink enough ... I think in this case, that is maybe what led to mine ... and not going to toilet when you need to, you hold on a lot. I think perhaps those two things do contribute to it a great deal"

17 Pregnancy, hormones, menstruation, tampons (irritation not infection), sex (aggravation)

18 The doctor "told me it was to do with sexual activity, so I presume that's what it was because things had changed in my life which I explained to her"

19 A highly moral discourse again; "I will be prepared to admit it's my own fault [laughter] 'cause I'm terrible, I just, coffee addict and . . I l just hardly every drink water ... I'm trying ... to do better" "I did have a really hot temperature ... I didn't know whether I was having hot flushes. I'm 44 [slight laughter] I thought, oh, maybe I might be having the change"

\section{0 "Not drinking enough"}

*Seven women were in symptoms score group, nine in empirical delayed group, and two each in symptoms score and mid-stream urine groups. We selected women for interview who had been asked to delay taking antibiotics to obtain their views on delay strategy. This would not apply to women in immediate empirical antibiotic group.

appropriate self care strategies when faced with symptoms, and so on) (table 4).

\section{DISCUSSION}

From this qualitative interview study we found that women with urinary tract infection want to avoid taking antibiotics and are open to alternative management strategies, including a delayed antibiotic prescription. They valued the opportunity to avoid the unwanted side effects associated with antibiotics and to allow "natural" healing of the body. For most, delayed prescribing was reassuring on two levels. Firstly, having a prescription waiting in the general practice reception was reassuring because it meant that they could collect the antibiotic should their symptoms worsen. Secondly, having a prescription available to them validated patients' experiences of their symptoms. There were a few negative experiences of delay, when being asked to "wait some more" served to undermine the legitimacy of their visit. Finally, our study indicates that women with and without previous experiences of urinary tract infection might not fully understand the causes or might draw on a theory of self blame and negligence, or both. Some women seemed to view antibiotics as necessary because they believed that they expedite the alleviation of symptoms.

\section{Results in context}

Urinary tract infections are common and are of concern to generalists (general practitioners, family doctors, urgent care) and specialists (including gynaecologists, urologists, and renal physicians). Little is available, however, on women's experiences and views of urinary tract infection and its management, especially the particular issue of alternative management strategies.

The strategy of antibiotic delay can be used in the management of sore throat and conjunctivitis ${ }^{16}$ and has been found to be acceptable to patients. ${ }^{27}$ The study of Everitt et al also indicated that patients will accept and encourage an alternative management strategy, including antibiotic delay. ${ }^{27}$ Increased information for and discussion with women attending for urinary tract infection is vital, however, if they are to better understand the rationale that underlies antibiotic delay. A full understanding might help to increase women's sense of validation and of being taken seriously.

Women might attribute urinary tract infection to issues of poor cleanliness and general "negligence." Lifestyles have been fully commercialised, ${ }^{28}$ and it is unsurprising to find that participants spoke in terms that suggested a (moral) duty to "consume a healthy lifestyle." It is important for general practitioners to be aware of them in the clinical encounter and be mindful of the consequences of such beliefs. For example, the cause of urinary tract infection might come to be construed as indicative of personal weakness. ${ }^{29}$ Women who tend to use a theory of self blame and negligence might be particularly vulnerable to feelings of not being taken seriously when their doctor proposes a strategy of no antibiotic or delayed antibiotic.

\section{Strengths and limitations}

Representation of thematic analysis can result in the de-contextualisation of speakers' words from the original sequence, which might misrepresent the intended meaning. We took care to analyse the participants' words in the broader context of the surrounding speech to ensure a fair interpretation of the meaning of the fragments reported here.

Women indicated some discomfort when asked to discuss the cause of urinary tract infection. It is difficult to know whether this reflected "limited knowledge" or whether the context of the interview might have caused 


\section{WHAT IS ALREADY KNOWN ON THIS TOPIC}

Patients' understanding of the causes of urinary tract infection and their views on and experiences of different management strategies are not well understood

The potential effectiveness of antibiotics for urinary tract infection needs to be balanced against wider issues such as side effects, antibiotic resistance, and general practitioners' workload

\section{WHAT THIS STUDY ADDS}

Women with urinary tract infection want to avoid taking antibiotic medication and are open to alternative management strategies, including a delayed antibiotic prescription

Clinicians need to acknowledge women's experiences before consulting (including attempts to self care), and if women are asked to delay taking antibiotics, the clinician must address the particular worries that women might have, and explain the rationale for not using antibiotics immediately could usefully explore the negotiation of delayed antibiotic prescribing and other options in situ.

\section{Implications for future research or clinical practice}

Patients' expectations do not necessarily revolve around healthcare professionals' prescription notepads. Rather, expectations are likely to centre on being understood and believed and in being helped to understand the rationale for alternative management strategies, such as antibiotic delay.

Some healthcare professionals might overestimate patients' demand for antibiotics. ${ }^{3031}$ And patients might also overestimate the desire of healthcare professionals to prescribe.

"We know that viral infections don't necessarily respond to antibiotics but I do think that there is a general feeling out there that a lot of GPs will just go yeah here's a course of antibiotics just to get you out of the door and move on to the next one" (patient 2)

Background expectations like these have potential to influence the consultation. If doctors recommend delayed antibiotics, the reasons must be clear. Equally, doctors need to be cautious in assuming that patients expect antibiotics. In our study, it seemed that the option to avoid the side effects of antibiotic treatment could lead to a sense of relief, and the opportunity to try other approaches was viewed positively. The next challenge is to establish whether and how these findings relate to clinical practice outside the context of a trial. A priority for exploration is whether there are advantages to offering an alternative to antibiotics when no antibiotic or a delayed antibiotic is recommended.

Overall, findings suggest that the particular strategy of delay could represent a happy medium for patients whereby they feel validated and reassured by the availability of a prescription, "just in case." A clear acknowledgement of a woman's triggers to consult is needed. If women are asked to delay taking antibiotics, the clinician must address the particular worries that women might have and explain the rationale for not prescribing antibiotics immediately.

We thank the practices that took part in the study and the patients who gave their time and shared their experiences and thoughts. The UTIS team comprises (in alphabetical order) C Hawke, J Lowes, K Martinson, MV Moore, MA Mullee, D Turner, and G Warner.

Contributors: PL had the original idea for the overall protocol and led the funding application. GML had the idea for the qualitative component of the study. All authors contributed to the development of the protocol and overall supervision of the study. ST, the trial coordinator, conducted the interviews with GML. GML and PL led the qualitative analysis and drafted the paper. All authors contributed to the writing of the final version of the paper. GML is guarantor.

Funding: This work was supported by the Health Technology Assessment programme grant reference: 97/14/06. The HTA had no involvement in the research process or writing of this article.

Competing interests: None declared.

Ethical approval: The study was approved by the local research ethics committee for Southampton and South West Hampshire (MREC/03/6/11) and informed consent was given by all patients.

Data sharing: No additional data available. 
1 Foxman B. Epidemiology of urinary tract infections: incidence, morbidity, and economic costs. Am J Med 2002;113:5-13.

2 Office of Population Censuses and Surveys (OPCS). Morbidity statistics from general practice: fourth national study 1991-1992. HMSO, 1994.

3 Milo G, Katchman EA, Paul M, Christiaens T, Baerheim A, Leibovici L. Duration of antibacterial treatment for uncomplicated urinary tract infection in women. Cochrane Database Syst Rev 2005;(2):CD004682.

4 Albert X, Huertas I, Pereiro I, Sanfélix J, Gosalbes V, Perrotta C. Antibiotics for preventing recurrent urinary tract infections in nonpregnant women. Cochrane Database Syst Rev 2004;(3):CD001209.

5 Fourcroy JL, Berner B, Chiang YK, Cramer M, Rowe L, Shore N. Efficacy and safety of a novel once-daily extended-release ciprofloxacin tablet formulation for treatment of uncomplicated urinary tract infection in women. Antimicrob Agents Chemother 2005;49:4137-43.

6 Ahmed M, Sloan JE, Clemente E. Clinical efficacy and safety of trimethoprim $\mathrm{HC} 1$ oral solution in the treatment of acute otitis media and urinary tract infection in children. Today's Therapeutic Trends 2001;19:63-76.

7 Henry DC, Nenad RC, Iravani A, Tice AD, Mansfield DL, Magner DJ, et al. Comparison of sparfloxacin and ciprofloxacin in the treatment of community-acquired acute uncomplicated urinary tract infection in women. Clin Ther 1999;21:966-81.

8 Leibovicki L, Wysenbeek A. Single dose antibiotic treatment for symptomatic urinary tract infections in women: a meta-analysis of randomised trials. Q J Med 1991;78:43-57.

9 DeAlleaume L, Tweed EM. When are empiric antibiotics appropriate for urinary tract infection symptoms? J Fam Pract 2006;55:338-42.

10 Mclsaac WJ, Low DE, Biringer A, Pimlott N, Evans M, Glazier R. The impact of empirical management of acute cystitis on unnecessary antibiotic use. Arch Intern Med 2002;162:600-5.

11 Lee JBL, Neild GH. Urinary tract infection. Medicine 2007;35:423-8.

12 Urinary tract infection. MeReC Bulletin 1995;6:29-32.

13 Avorn J, Monane M. Reduction of bacteriuria and pyuria after ingestion of cranberry juice. JAMA 1994;271:751-4.

14 Goessens WH, Verbrugh HA. Antibiotic resistance: epidemiological developments and preventive measures. Ned Tijdschr Geneeskd 2007;151:748-52.

15 Tam VH, Louie A, Fritsche TR, Deziel M, Liu W, Brown DL, et al. Impact of drug-exposure intensity and duration of therapy on the emergence of Staphylococcus aureus resistance to a quinolone antimicrobial. $)$ Infect Dis 2007;195:1818-27.

16 Little PS, Williamson I, Warner G, Gould C, Gantley M, Kinmonth AL. An open randomised trial of prescribing strategies for sore throat. BMJ 1997;314:722-7.
17 Little PS, Gould C, Williamson I, Warner G, Gantley M, Kinmonth AL. Reattendance and complications in a randomised trial of prescribing strategies for sore throat: the medicalising effect of prescribing antibiotics. BMJ 1997;315:350-2.

18 McNulty CA, Richards J, Livermore DM, Little P, Charlett A, Freeman E, et al. Clinical relevance of laboratory-reported antibiotic resistance in acute uncomplicated urinary tract infection in primary care. J Antimicrob Chemother 2006;58:1000-8.

19 Hart C. Antibiotic resistance: an increasing problem? BMJ 1998;316:1255-6.

20 Arason V, Kristinsson K, Sigurdsson J, Stefansdottir G, Molstad S, Gudmundsson S. Do antimicrobials increase the rate of penicillin resistant pneumococci in children? Cross sectional prevalence study. BMJ 1996;313:387-91.

21 Christiaens T, DeMeyere M, Verschraegen G, Peersman W, Heytens S, DeMaeseneer J. Randomised controlled trial of nitrofurantoin versus placebo in the treatment of uncomplicated urinary tract infection in adult women. BrJ Gen Pract 2002;52:729-34.

22 Little P, Moore M, Turner S, Rumsby K, Warner G, Lowes A, et al. Effectiveness of five different approaches in the management of urinary tract infection: a randomised controlled trial. $B M$ 2010;doi:10.1136/bmj.c199.

23 Silverman D. Interpreting qualitative data: methods for analysing talk, text and interaction. Sage, 2001.

24 Patton MQ. Qualitative research and evaluation methods. 3rd ed. Sage, 2002.

25 Barbour R, members of WeReN. Acquiring qualitative skills for primary care research. Review and reflections on three-stage workshop. Part 2: analysing interview data. Fam Pract 2000;17:83-9.

26 Leydon GM, Turner S, Smith H, Little P. The journey from self-care to GP care: a qualitative interview study of women presenting with symptoms of urinary tract infection. Br J Gen Pract 2009;59:e219-25.

27 Everitt H, Kumar S, Little P. A qualitative study of patients' perceptions of acute infective conjunctivitis. Br J Gen Pract 2003;53:36-41.

28 Nettleton S. The sociology of health and illness. Polity Press, 1995.

29 Blaxter M. Whose fault is it? People's own conceptions of the reasons for health inequalities. Soc Sci Med 1997;44:747-56.

30 Stivers T. Participating in decisions about treatment: overt parent pressure for antibiotic medication in pediatric encounters. Soc Sci Med 2002;54:1111-30.

31 Butler CC, Kinnersley P, Prout H, Rollnick S, Edwards A, Elwyn G. Antibiotics and shared decision-making in primary care. / Antimicrob Chemother 2001;48:435-40.

Accepted: 23 October 2009 